\title{
Analysis and modeling of a modular ISOP Full Bridge based converter with input filter
}

\author{
P. Zumel, E. Oña, C. Fernandez, M. Sanz, A. Lazaro, A. Barrado \\ GSEP, Power Electronics System Group \\ Carlos III University of Madrid \\ Leganes, Spain
}

\begin{abstract}
This work presents a modular architecture based on the input series output parallel (ISOP) connection of Full Bridge Phase Shifted power converters with a common input filter. The modeling of the converter architecture taking into account the effect of the common input filter is the previous step to the controller design in order to ensure a proper regulation of external converter quantities, such as output voltage, and internal quantities, such as input voltages. In this paper a small signal model of an ISOP Full Bridge Phase Shifted converter taking into consideration the input filter is developed. Simulation and experimental results validate the theoretical predictions.
\end{abstract}

Keywords-ISOP, full-bridge, modular converter, converter grouping

\section{INTRODUCTION}

The connection of power converters in modular architectures or multicell converters has been used in the last years due to the technological and economic advantages [1][11]. In a modular ISOP (input series output parallel) converter the input voltage and the output current are distributed among the modules. Advantages of ISOP connection are the capability to handle high voltages with low voltages devices which exhibit better figures of merit than devices rated for higher voltages, resulting in lower losses and higher switching frequencies. Modular converters have been used in Solid State Transformers, microgrids, etc.

\author{
A. Vazquez, D.G. Lamar \\ Electronic Power Supply Systems Group (SEA) \\ University of Oviedo \\ 33204, Gijon, Spain
}

Besides the control of the output quantities, e.g. output voltages, modular converters require the control of inner quantities, i.e., input voltages or output currents in each module in the case of ISOP connection. The control of modular converters presents several challenges [2]-[11]. If all modules are exactly equal, all electrical quantities are evenly distributed among them, and no especial care about voltages and current sharing has to be taken into account. However, in mass production, considering the component tolerances, the modules may differ one from the other, and the control has to ensure a proper distribution of voltages and current in order to avoid oversizing of the converter modules.

Previous works have dealt with full bridge ISOP connection [7][9]. A strategy based on decoupling control loops is proposed in [7] applied to ISOP full bridge converters without input filter. In this work the modeling is reviewed and the effect of the input filter is considered, which can change dramatically the behavior of the modular converter. In the ISOP connection, the input filter couples the dynamic response of the modules in such a way that the conventional decoupling techniques have to be reviewed. In this paper, the analytical model of the ISOP modular converter with common input filter is presented. Simulations are performed to illustrate theoretical predictions and control strategies. Finally, an experimental setup has been used to validate the obtained model.

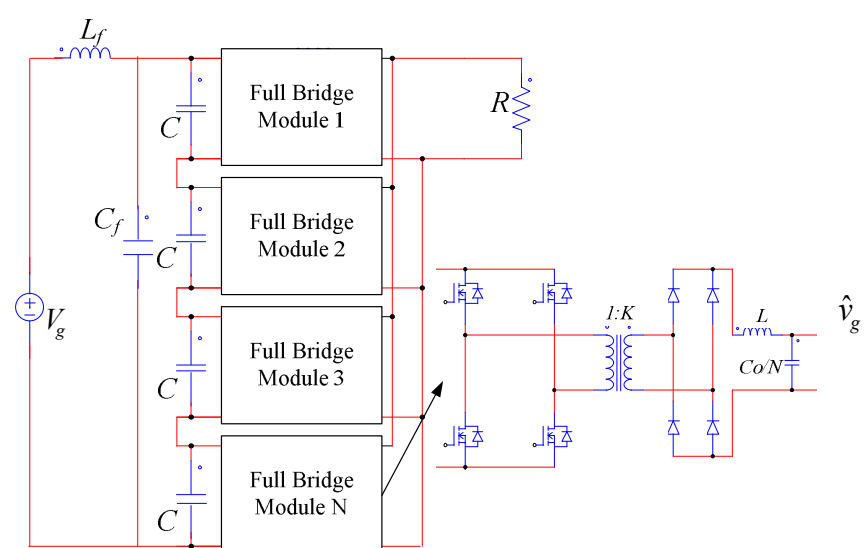

(a)

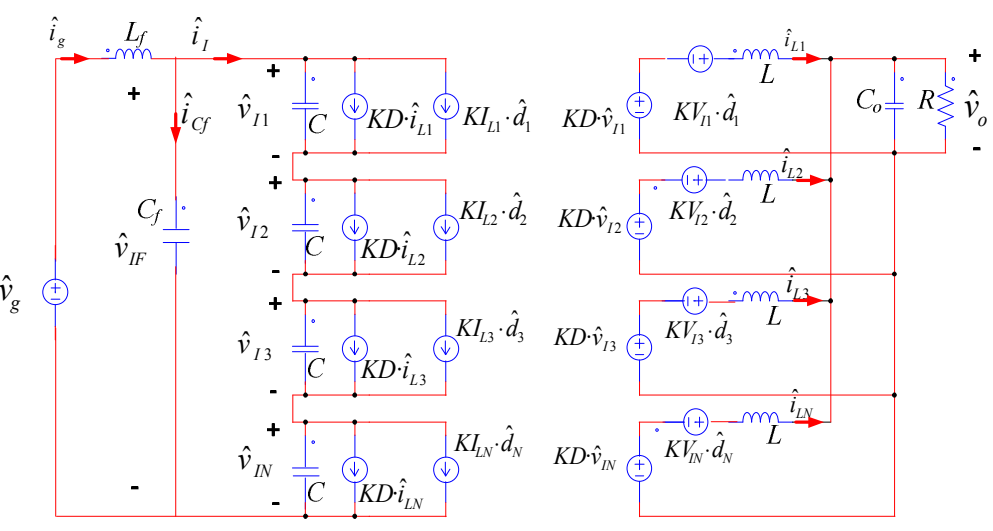

(b)

Fig. 1. a) ISOP modular converter based on Full Brdige modules with input filter, b)Small signal averaged model of ISOP with input filter

This work has been partially supported by the Ministry of Economy and Competitiveness and FEDER funds, through the research projects ELECTRICAR-AG- (DPI2014-53685-C2- 1-R), Consolider RUE CSD200900046 and DPI2013-47176-C2-2-R 


\section{SMALL SIGNAL MODEL OF AN ISOP MODULAR FULL BRIDGE CONVERTER}

The small signal model of the modular architecture is based on the circuit presented in Fig. 1. The modular converter is based on Full Bridge Phase Shift converters. Each module has been replaced by its averaged and linearized circuit. The derivation of the small signal model is detailed in Appendix.

The control strategy for the proposed ISOP modular converter with $N$ modules is based on the control of the total output voltage $\hat{v}_{o}$ and the input voltages of $N-1$ modules $\hat{v}_{I 1}, \ldots, \hat{v}_{I(N-1)}$. Therefore, the goal of the modeling analysis is to obtain the expressions relating $\hat{v}_{I 1}, \ldots, \hat{v}_{I N}$ and $\hat{v}_{o}$ with the control signals $\hat{d}_{1}, \ldots, \hat{d}_{N}$ (normalized phase shifts or duty cycles) considering the circuits parameters of the modular converter. In order to obtain a model taking into account the effect of parasitic components, impedances have been considered for each element: $Z_{L f}$ for inductor filter $L_{f}, Z_{C f}$ for capacitor filter $C_{f}, Z_{C}$ for input capacitor $C, Z_{L}$ for output inductor $L$ and $Z_{O}$ for the output capacitor $C_{O}$ in parallel with the load resistance $R$.

The assumptions in the model derivation are the following: a) all modules have the same characteristics (inductance, resistance and capacitance values); b) all modules are in the same operating point (same input voltage $\frac{V_{G}}{N}$, output current $I_{L}$, and duty cycle $D$ ).

\section{A. Small signal model without input filter and decoupling control loops}

From expressions derived in Appendix, when no input filter $\left(Z_{L f}=0\right)$ is used, output voltage $\hat{v}_{o}$ and input voltage $\hat{v}_{I j}$ of module $\mathrm{j}$ become respectively ( 1 ) and (2). It should be noted that $Z_{L}, Z_{C}, Z_{O}, G_{v d}$ and $A$ are functions of the complex variable "s", while $\mathrm{K}$ (transformation ratio), $V_{G}$ (average input voltage) and $\mathrm{N}$ are constants.

$$
\begin{aligned}
& \hat{v}_{o}=\frac{K V_{G}}{N+\frac{Z_{L}}{Z_{O}}} \frac{\left(\hat{d}_{1}+\cdots+\hat{d}_{N}\right)}{N}=G_{v d} \frac{\left(\hat{d}_{1}+\cdots+\hat{d}_{N}\right)}{N} \\
& \hat{v}_{I j}=-A \frac{\left(\hat{d}_{1}+\cdots+\hat{d}_{N}\right)}{N}+A \hat{d}_{j}= \\
& =-\frac{A}{N}\left(\hat{d}_{1}+\cdots-(N-1) \hat{d}_{j}+\cdots+\hat{d}_{N}\right)
\end{aligned}
$$

Where $A$ is given by ( 3 ).

$$
A=-\frac{K\left(\frac{V_{O}}{N R}+\frac{D K V_{G}}{N Z_{L}}\right)}{\frac{1}{Z_{C}}+\frac{D^{2} K^{2}}{Z_{L}}}
$$

The modular converter can be described by the matrix equation (4).

$$
\left[\begin{array}{c}
\hat{v}_{I 1} \\
\vdots \\
\hat{v}_{I N-1} \\
\hat{v}_{o}
\end{array}\right]=\left[\begin{array}{cccc}
\frac{N-1}{N} A & \frac{-A}{N} & \ldots & \frac{-A}{N} \\
\vdots & \ddots & & \vdots \\
-A & & \frac{N-1}{N} A & \frac{-A}{N} \\
\frac{N}{G_{V d}} & G_{V d} & G_{V d} & G_{V d}
\end{array}\right]\left[\begin{array}{c}
\hat{d}_{1} \\
\vdots \\
\hat{d}_{N-1} \\
\hat{d}_{N}
\end{array}\right]
$$

Expression ( 4) shows that all controlled quantities $\left(\hat{v}_{o}\right.$ and $\mathrm{N}-1$ inputs voltages $\hat{v}_{I j}$ ) depend on all control variables $\left(\hat{d}_{1}, \cdots, \hat{d}_{N}\right)$, resulting in a MIMO (multiple input multiple output) system. A simple strategy to simplify the system and to obtain several independent SISO (single input single output) systems it based on the fact that the controlled quantities $\left(\hat{v}_{I 1}, \ldots, \hat{v}_{I(N-1)}\right.$ and $\left.\hat{v}_{o}\right)$ depend on different linear combinations of the control variables $\left(\hat{d}_{1}, \ldots, \hat{d}_{N}\right)$, as shown in ( 1 ) and (2). A change of variables can be made in order to replace each linear combination of $\hat{d}_{j}$ by a new control quantity $\hat{x}_{j}$, obtaining a new description in which each controlled quantity depends on a single control signal. This decoupling transformation was first proposed in [7] and was also used in [12][13]. The new system can be described with equations ( 5) and (6).

$$
\begin{gathered}
{\left[\begin{array}{c}
\hat{v}_{I 1} \\
\vdots \\
\hat{v}_{I N-1} \\
\hat{v}_{o}
\end{array}\right]=\left[\begin{array}{ccccc}
-A & 0 & & \ldots & 0 \\
\vdots & \ddots & & \vdots \\
0 & \ldots & -A & 0 \\
0 & 0 & 0 & N G_{V d}
\end{array}\right]\left[\begin{array}{c}
\hat{x}_{1} \\
\vdots \\
\hat{x}_{N-1} \\
\hat{x}_{N}
\end{array}\right]} \\
{\left[\begin{array}{c}
\hat{d}_{1} \\
\vdots \\
\hat{d}_{N-1} \\
\hat{d}_{N}
\end{array}\right]=\left[\begin{array}{cccccc}
-1 & 0 & 0 & \ldots & 0 & 1 \\
0 & -1 & 0 & \ldots & 0 & 1 \\
\vdots & \vdots & \vdots & \vdots & \vdots & \vdots \\
0 & 0 & 0 & \ldots & -1 & 1 \\
1 & 1 & 1 & \ldots & 1 & 1
\end{array}\right]\left[\begin{array}{c}
\hat{x}_{1} \\
\hat{x}_{2} \\
\vdots \\
\hat{x}_{N-1} \\
\hat{x}_{N}
\end{array}\right]}
\end{gathered}
$$

Expressions ( 5) and ( 6) allow to implement $\mathrm{N}$ independent control loops to control the total output voltage and N-1 input voltages, as described in Fig. 2. Expression ( 5) defines the plant to be considered for the compensator calculation, while expression ( 6) defines the translation from the fictitious variables $\hat{x}_{j}$ calculated by compensator $\mathrm{C} 1(\mathrm{~s})$ and $\mathrm{C} 2(\mathrm{~s})$ and the real variables $\hat{d}_{j}$ to be applied to the actual converter. This control scheme is shown in Fig. 3.

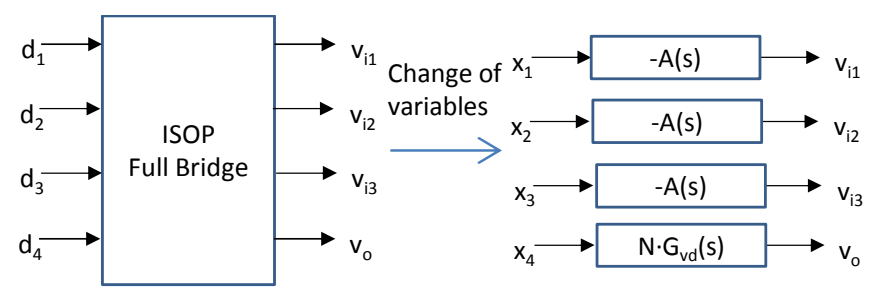

Fig. 2. Control strategy to decoupled control loop in a ISOP modular converter without input filter. 


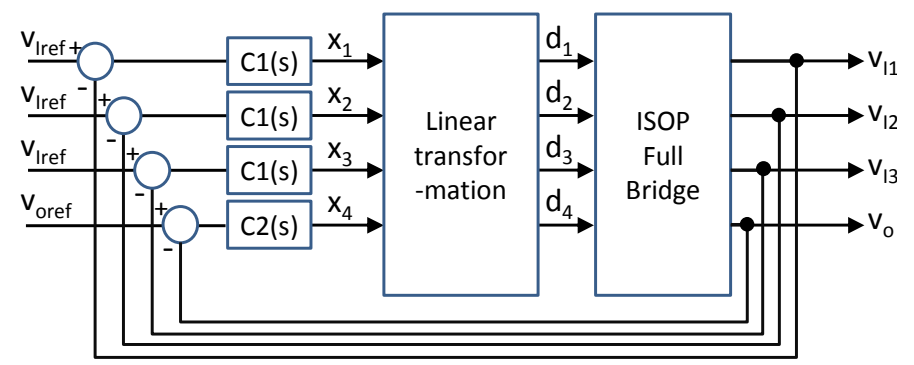

Fig. 3. Control strategy to decoupled control loop in a ISOP modular converter without input filter.

The condition to apply this strategy is that each controlled quantity depends on a linear combination of the control variables, and all these linear combinations are independent. If this condition is fulfilled, the implementation of the control system is easy since the generation of the duty cycle signals from the values of $\hat{x}_{1}, \ldots, \hat{x}_{N}$ calculated in the control loops is carried out by means simple algebraic operations (6).

\section{B. Small signal model considering input filter}

From the derivations of Appendix, the expression of the output voltage and input voltage of module $\mathrm{j}$ are (7) and (8) respectively.

$$
\begin{gathered}
\hat{v}_{o}=G_{V d F} \frac{\left(\hat{d}_{1}+\cdots+\hat{d}_{N}\right)}{N} \\
\hat{v}_{I j}=A_{1} \frac{\left(\hat{d}_{1}+\cdots+\hat{d}_{N}\right)}{N}-A_{2} \hat{d}_{j}
\end{gathered}
$$

It should be noted that in this case input voltage $\hat{v}_{I j}$ is not a linear combination of $\hat{d}_{j}$, since transfer functions $A_{1}$ and $A_{2}$ are not proportional. $G_{V d F}, A_{1}$ and $A_{2}$ are functions of "s".

The new system can be written in a matrix form (9).

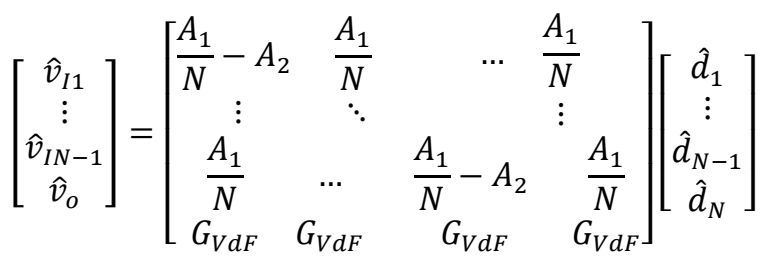

Due to the effect of the input filter on the input voltage transfer function ( 8 ) it is not possible to apply a simple transformation to the original MIMO system to obtain N SISO systems. The condition referred in the previous paragraphs is not met, i.e., input voltages do not depend on linear combinations of control signals $\hat{d}_{j}$, and therefore expression ( 6 ) is not valid in this case. In Fig. 4 frequency response of input voltage $\hat{v}_{I 1} / \hat{d}_{1}$ and $\hat{v}_{I 2} / \hat{d}_{1}$ are plotted. In this example the following parameters has been used: $V_{G}=2600 \mathrm{~V}, N=4$, $K=1.48, L_{f}=39.5 \mathrm{mH}, C_{f}=1 \mathrm{mF}, L=420 \mu \mathrm{H}, C_{o}=1.32 \mathrm{mF}$, $R=2.34 \Omega$. Parasitic resistances have been considered in all elements. It should be noted that in the case without input filter, $\hat{v}_{I 1} / \hat{d}_{1}$ and $\hat{v}_{I 2} / \hat{d}_{1}$ are proportional, i.e., there is an offset in the magnitude plot and a constant difference of phase equal to $180^{\circ}$.
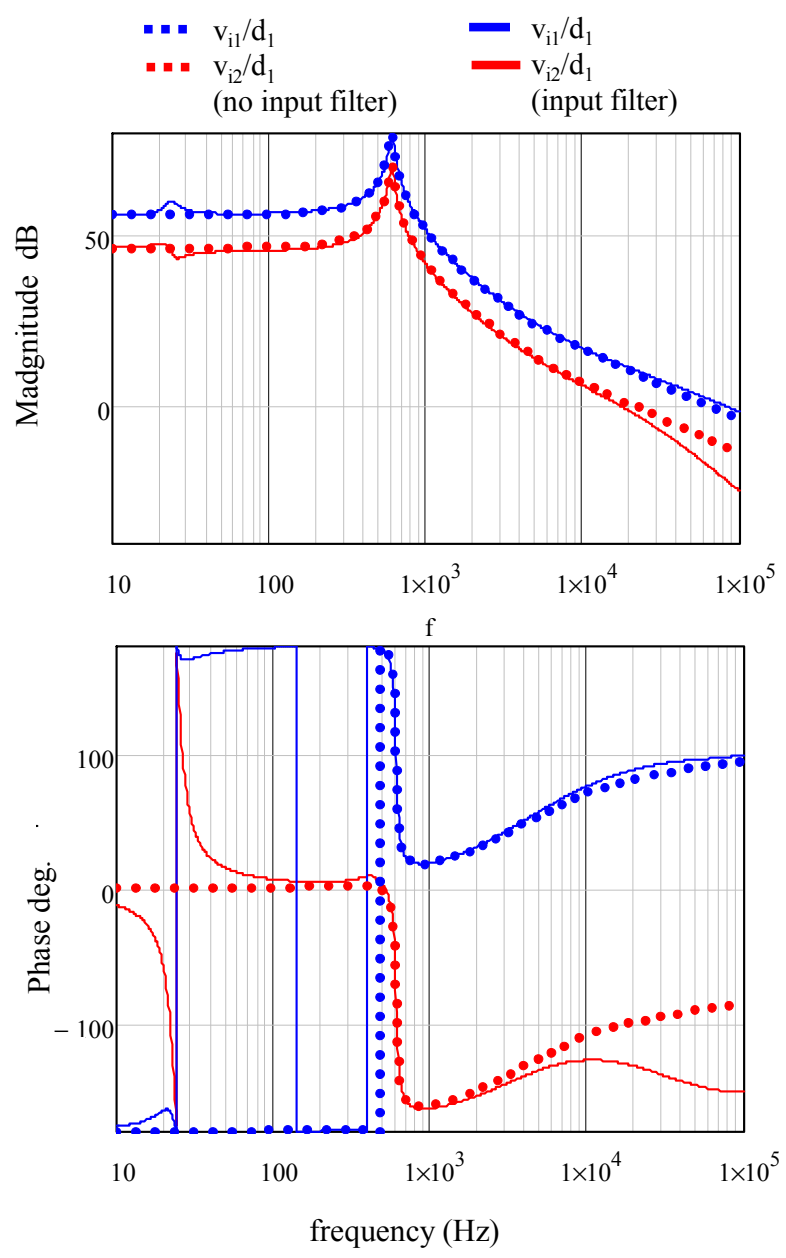

Fig. 4. Frequency response of the input voltage of module $1 \hat{v}_{I 1} / \hat{d}_{1}$ and input voltage of module $2 \hat{v}_{I 2} / \hat{d}_{1}$, with input filter (solid lines) and without input filter (dashed lines)

A similar change of variable can be applied to (9), obtaining expressions ( 10) and (11). In order to illustrate this calculation step, these expressions have been particularized for $\mathrm{N}=4$. Expression ( 11 ) is used to generate the actual control signals $\hat{d}_{1}, \ldots, \hat{d}_{N}$ to be applied to the actual converter. The main limitation of this approach is the physical implementation of this algorithm due to two reasons: 1) it is required to obtain $\frac{A_{1}}{A_{2}}$, which can be complex because of its dependence on parasitic components; 2) this transfer function is a filter with a high order in the numerator and denominator.

$$
\left[\begin{array}{c}
\hat{v}_{I 1} \\
\hat{v}_{I 2} \\
\hat{v}_{I 3} \\
\hat{v}_{o}
\end{array}\right]=\left[\begin{array}{cccc}
A_{2} & 0 & 0 & 0 \\
0 & A_{2} & 0 & 0 \\
0 & 0 & A_{2} & 0 \\
0 & 0 & 0 & G_{V d}
\end{array}\right]\left[\begin{array}{l}
\hat{x}_{1} \\
\hat{x}_{2} \\
\hat{x}_{3} \\
\hat{x}_{4}
\end{array}\right]
$$




$$
\left[\begin{array}{l}
\hat{d}_{1} \\
\hat{d}_{2} \\
\hat{d}_{3} \\
\hat{d}_{4}
\end{array}\right]=\left[\begin{array}{cccc}
-1 & 0 & 0 & \frac{A_{1}}{4 A_{2}} \\
0 & -1 & 0 & \frac{A_{1}}{4 A_{2}} \\
0 & 0 & -1 & \frac{A_{1}}{4 A_{2}} \\
1 & 1 & 1 & 1-\frac{3 A_{1}}{4 A_{2}}
\end{array}\right]\left[\begin{array}{l}
\hat{x}_{1} \\
\hat{x}_{2} \\
\hat{x}_{3} \\
\hat{x}_{4}
\end{array}\right]
$$

The input filter has an influence on the overall modular converter similar to the case of a single module. When the output voltage is analyzed (Fig. 5), the effect of the input filter in the frequency response is similar to the classical buck converter with input filter [14]. While the magnitude plot is not very significantly affected, the phase exhibits a change of $360^{\circ}$ at the natural frequency of the input filter. Parasitic components or additional damping circuits affect to this response.
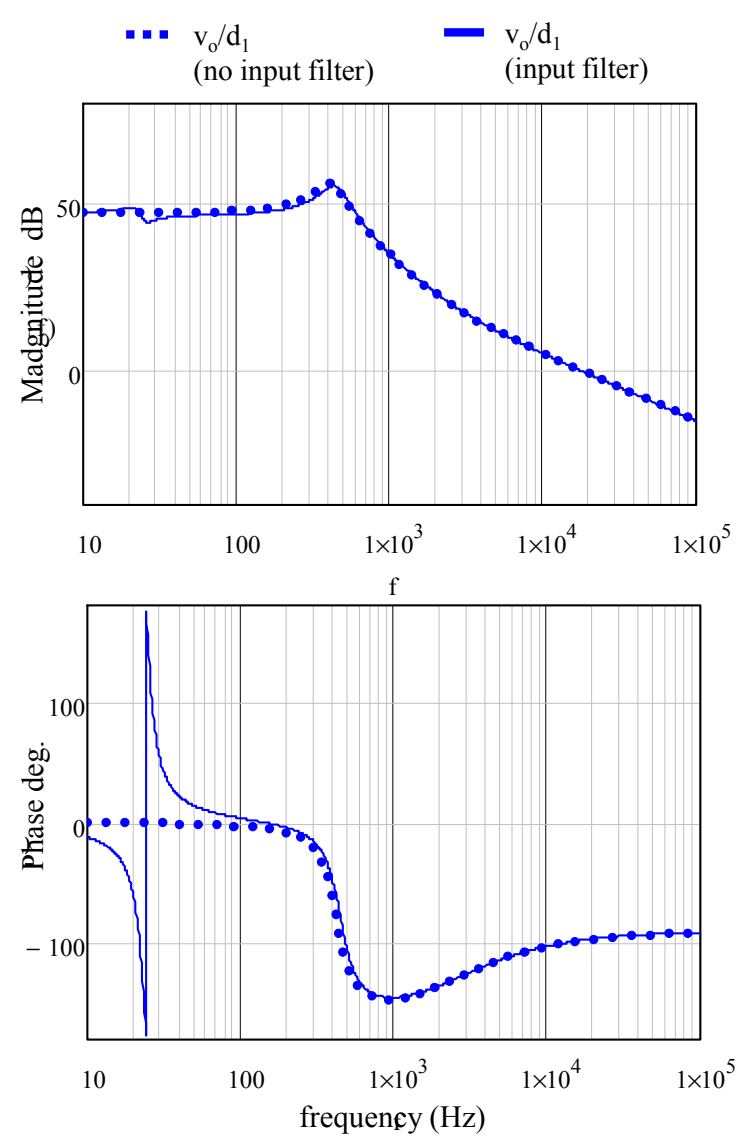

Fig. 5. Frequency response of the output $\hat{v}_{o}$ respect to $\hat{d}_{1}$ with input filter (solid line) and without input filter (dashed line).

As it has been shown, the decoupling strategy based on ( 6) cannot be directly used in the presence of input filter. When the input filter is used several options can be considered in order to overcome the effect on the control loops of the input filter: a) generate a decoupling strategy including transfer function $\frac{A_{1}}{A_{2}}$ in the control variable transformation, which implies complex transformation and additional study to ensure the feasibility of the calculations; b) design the controller for cross over frequencies under the frequency range where the filter has influence; c) applying damping techniques to the filter in order to overcome the influence in the control transfer functions.

(a)

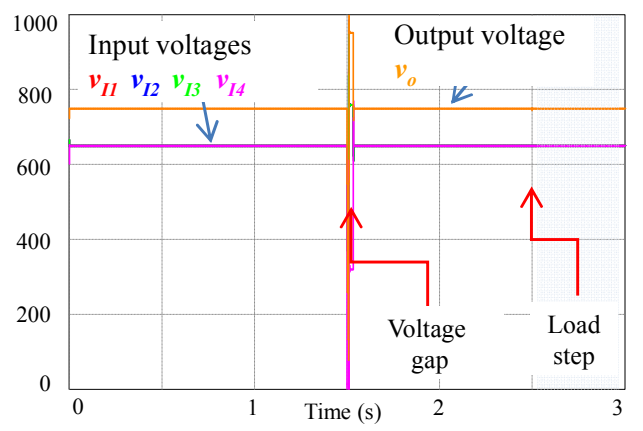

(b)
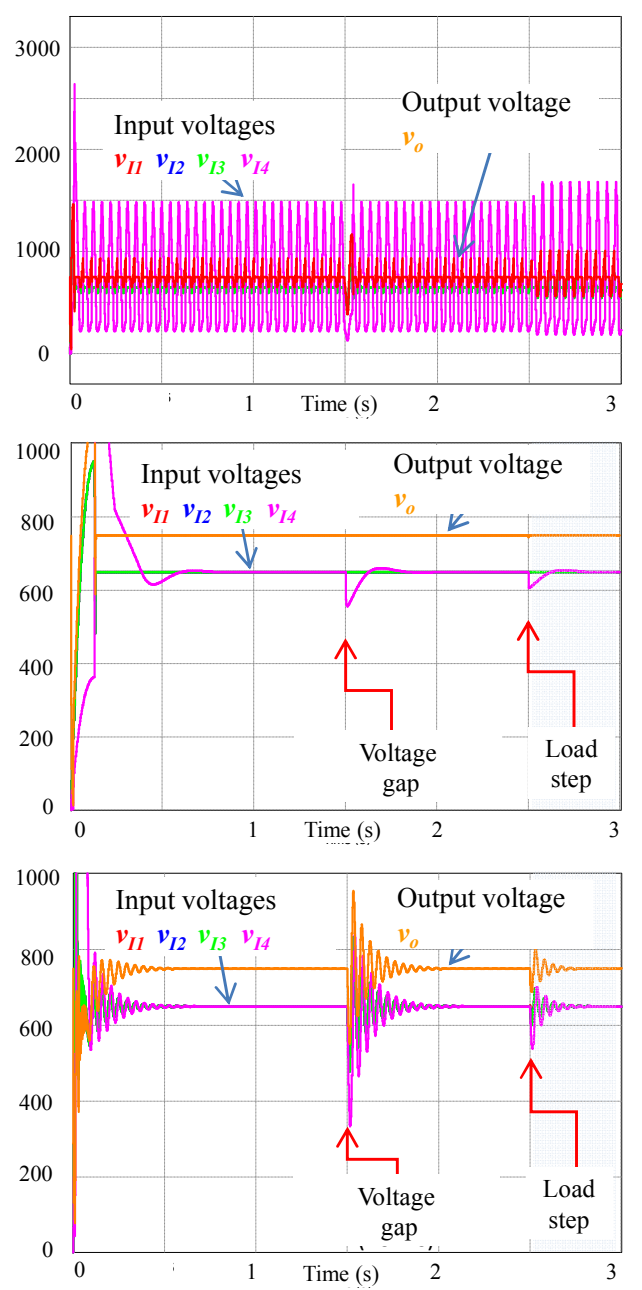

Fig. 6. Simulations of a four module ISOP modular converter

In the two last approaches, the original decoupling technique can be applied, since the controlled variable can be written as a linear combination of the control variables

These two possibilities have been simulated to illustrate this approach. Circuit parameters correspond to the example 
described in section II.b. In the time simulation input voltage gaps have been included in order to assess the performance of the input filter. Results are displayed in Fig. 6. A first test (Fig. $6 a)$ is performed to the modular converter is without input filter and using the control strategy described in Fig. 3. The same regulators have been used in Fig. $6 \mathrm{~b}$ but now there is an input filter, resulting in an unstable operation. In Fig. 6c the input filter has been damped, and the same controller than in the reference situation has been used. Finally, in Fig. 6d the regulators are designed with cross-over frequencies below the resonance frequency of the input filter.

\section{EXPERIMENTAL RESULTS}

In order to validate the small signal model, an experimental setup has been used to measure the transfer functions of input voltages and output voltages. In the laboratory, a set of three modules have been used in ISOP connection. Total input voltage is $50 \mathrm{~V}$, output voltage $100 \mathrm{~V}$, transformer ratio $\mathrm{K}=10$, $L_{f}=8 \mathrm{mH}, C_{f}=440 \mu \mathrm{F}, C=1720 \mu \mathrm{H}, L=337 \mu \mathrm{H}, C_{o}=3 \times 22 \mu \mathrm{F}$, $\mathrm{R}=30 \Omega$.

The aim of the lab test is to validate the relationship among the transfer functions and the influence of the input filter. Three measurement setups have been considered: modular converter without input filter, modular converter with input filter and modular converter with the input filter and an additional parallel R-C damping branch. The modular converter operates in open loop, controlled by an FPGA. The duty cycles of two modules are constant, while in the third module the duty cycle is introduced by an analog to digital converter. The duty cycle signal is the connected to the oscillator of the Frequency Response Analyzer. The probe gain has not been corrected. Frequency response measurements are shown in Fig. 7, Fig. 8 and Fig. 9, corresponding to systems without filter, system with input filter and system with damped input filter, respectively.

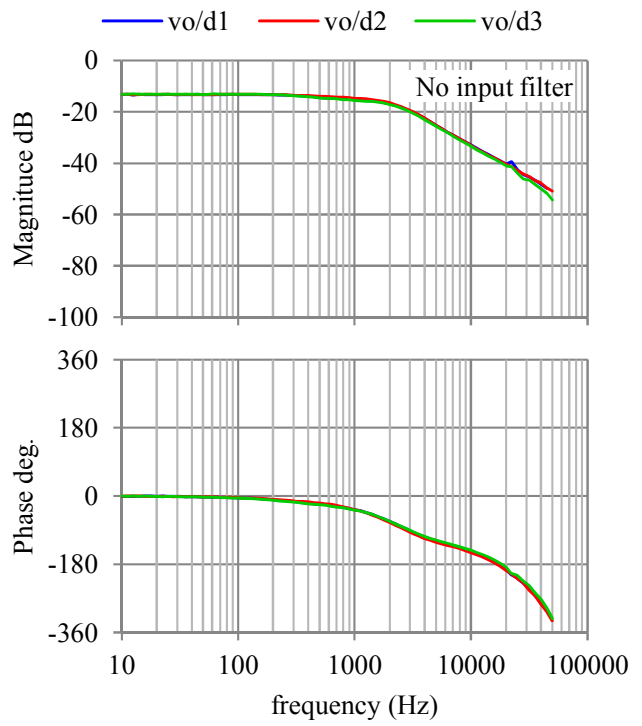

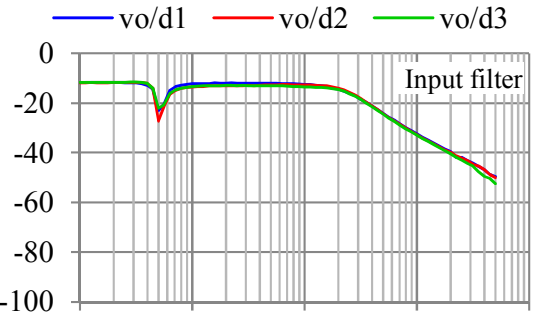
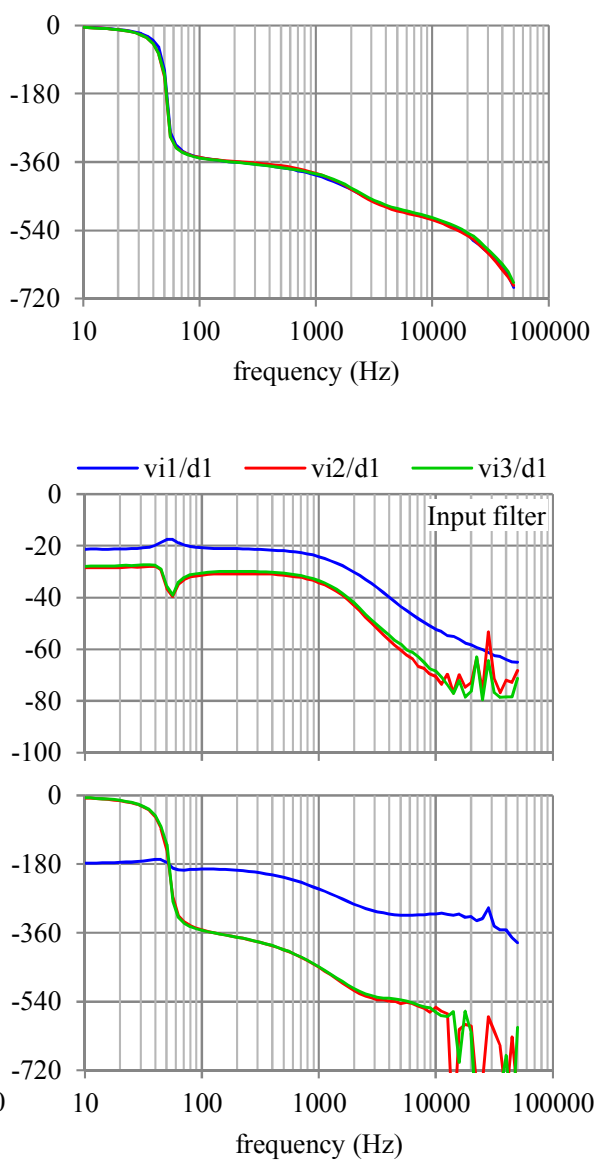

Fig. 8. Transfer functions with input filter
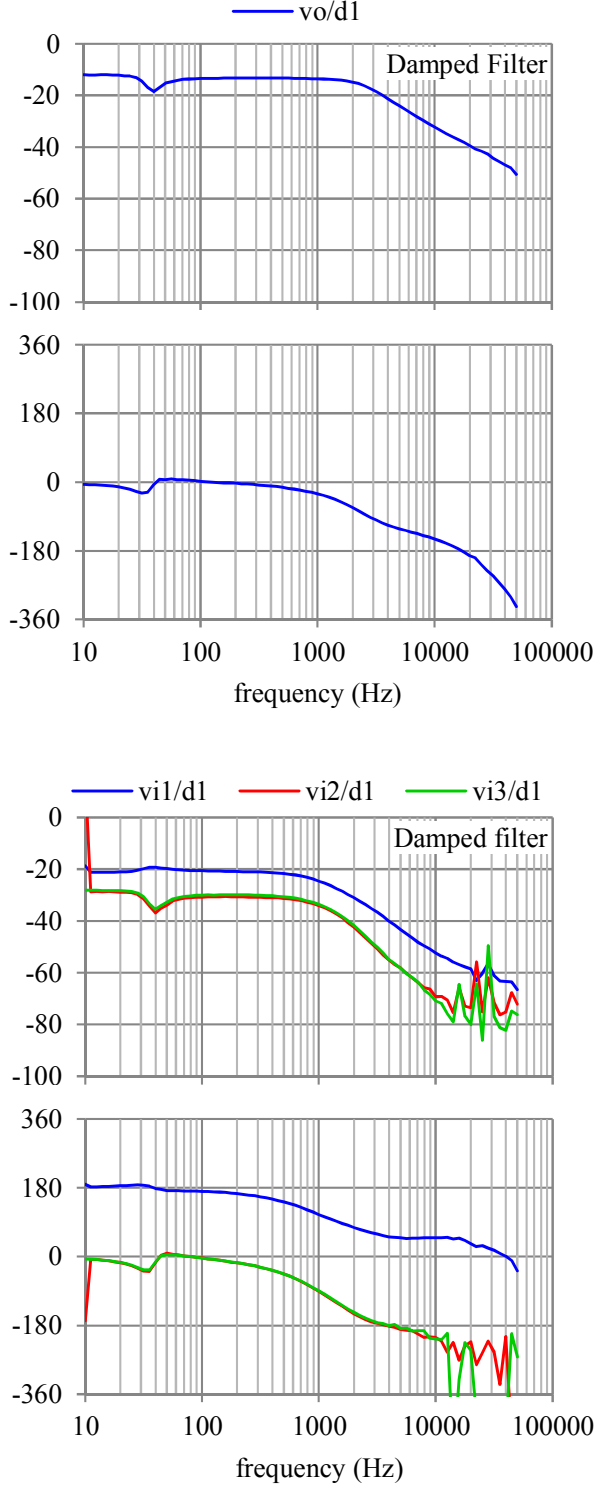

Fig. 9. Trasnfer function with damped input filter

Fig. 7. Transfer functions without input filter 
First, the relationships among the transfer functions can be considered. From the point of view of output voltage $\hat{v}_{o}$, it is important to check if it depends on the same way on the three duty cycles $\hat{d}_{j}$. It is shown in 0 and Fig. 8 , agreeing with the described characteristic. In the case of input voltages it is important to check that the transfer function of the input voltage of the modules with the non perturbed duty cycle $\left(\hat{v}_{I 2} / \hat{d}_{1}\right.$ and $\left.\hat{v}_{I 3} / \hat{d}_{1}\right)$ are similar, as it can be seen in Fig. 7, Fig. 8, and Fig. 9.

Second, the influence of the input filter can be analyzed. In the case of output voltage, when the input filter is used, the phase of the transfer function $\hat{v}_{o} / \hat{d}_{1}$ decreases $360^{\circ}$ due to the input filter, as in the classical buck converter with input filter [14], degrading the dynamic performance of the system. It should be noted that the effective resonance frequency of the input filter is not only defined by the filter components $L_{f}$ and $C_{f}$, but also by the series equivalent of the input capacitors $C$. If only $L_{f}$ and $C_{f}$ are considered, the resonance frequency is 85 Hz. However, if the equivalent of the input capacitors (1720 $\mu \mathrm{F} / 3$ ) is considered in parallel with $C_{f}$, the resulting resonance frequency is $56 \mathrm{~Hz}$, agreeing with experimental measurements. From the point of view of input voltages, it should be noted that due to the presence of input filter, $\hat{v}_{I 2} / \hat{d}_{1}$ and $\hat{v}_{I 1} / \hat{d}_{1}$ are not proportional (constant difference in magnitude, and $180^{\circ}$ or $0^{\circ}$ difference in phase). Consequently, decoupling technique based on linear transformation ( 6) would not obtain good results.

In Fig. 9 the frequency response of the system is measured using an additional damping R-C branch in parallel with $C_{f}$. From the point of view of output voltage it is now possible to design a compensator above the input filter resonance frequency. From the point of view of input voltages, even if it is not the ideal situation, transfer functions are compatible with the decoupling technique to design control loops.

Finally in 0 measurements have been compared with the theoretical models. In this case, parasitic components have been estimated from prototype frequency response. Modulator delay has not been considered in the theoretical model, while the gains of the probe, modulator and analog to digital converter have been taken into account in the comparison. It should be noted that the agreement between theory and measurements is extended to a significant frequency range.

\section{CONCLUSIONS}

In this paper, the effect of the input filter in a modular ISOP architecture based on Full Bridge Phase Shifted converters has been analyzed.

A small signal model has been obtained showing the importance of the input filter in the control strategy, from the point of view of the regulation output voltage and the distribution of the input voltage in each module. Classical decoupling techniques for the control of modular converter have to be carefully considered when using input filter, since an additional coupling among the modules appears. Simple strategies to overcome the effect of the input filter can be applied, as filter damping or slow controller design.

Frequency response measurements have been performed over a prototype of three modules. The influence on the output voltage and input voltages of the input filter (undamped and damped) has been analyzed, showing a good agreement between the theoretical model and experimental measurements.

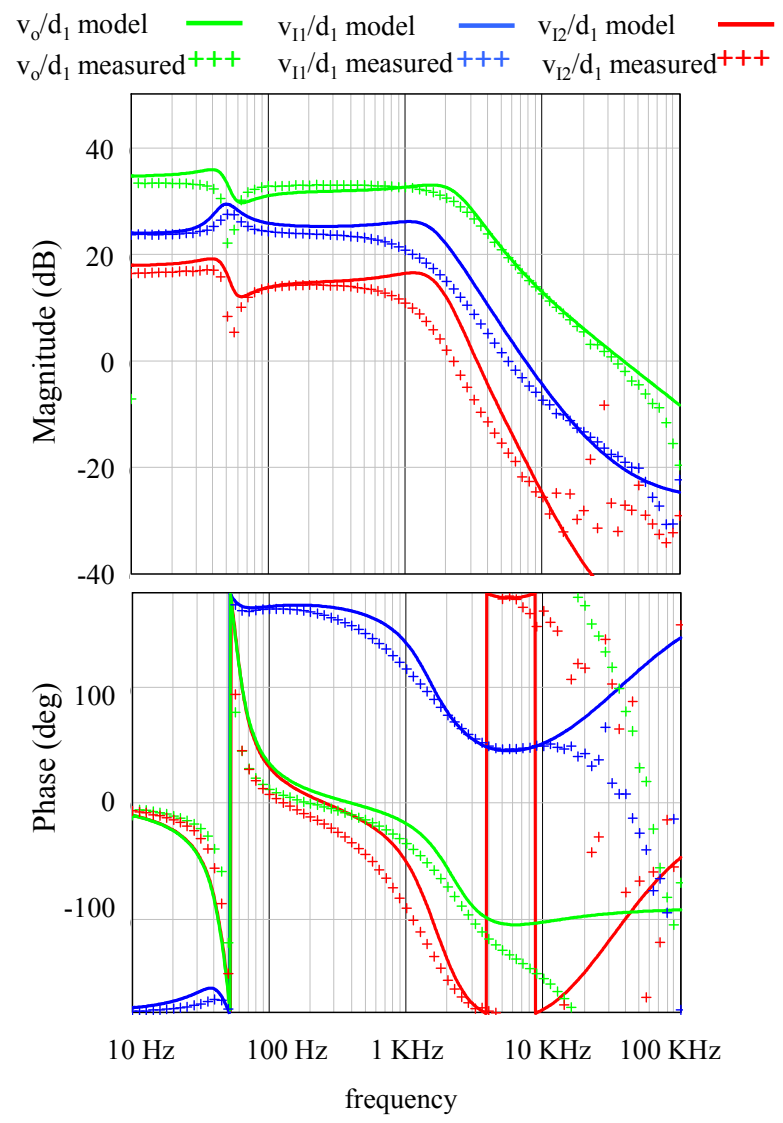

Fig. 10. Theoretical and measured trasnfer function comparison.

\section{APPENDIX: SMALL SIGNAL MODEL DERIVATION}

\section{A. Input current and output voltage expression derivation}

From the average circuit model of Fig. 1 and linearizing the response of the circuit, the output voltage $\hat{v}_{o}$ can be expressed as:

$$
\begin{gathered}
\hat{v}_{o}=\frac{K\left(\frac{V_{G}}{N}\left(\hat{d}_{1}+\cdots+\hat{d}_{N}\right)+D\left(\hat{v}_{I 1}+\cdots+\hat{v}_{I N}\right)\right) \frac{1}{z_{L}}}{\frac{N}{z_{L}}+\frac{1}{z_{O}}} \\
\hat{v}_{O}=\frac{K\left(\frac{V_{G}}{N}\left(\hat{d}_{1}+\cdots+\hat{d}_{N}\right)+D \hat{v}_{I F}\right)}{N+\frac{Z_{L}}{z_{O}}}
\end{gathered}
$$


Where $K$ is the turns ratio, $V_{G}$ is the total input voltage, $\mathrm{D}$ is the nominal duty cycle, $Z_{L}$ is the impedance of the output inductor (including parasitics), $Z_{O}$ is the output impedance (output capacitor in parallel with load), $\hat{d}_{i}$ is the duty cycle of each module, $\hat{v}_{I i}$ is the input voltage of a given module and $\hat{v}_{I F}$ is the input voltage of the modular converter after the input filter. In this expression, $\hat{v}_{I F}$ is not known, since it depends on the input current $\hat{l}_{I}$ and the filter impedance.

The input current $\hat{l}_{I}$ can be derived considering the input node of each converter. For the converter j, expression ( 14) corresponds to the input node.

$$
\hat{\imath}_{I}=\hat{\imath}_{C j}+K\left(D \cdot \hat{\imath}_{L j}+I_{L} \cdot \widehat{d}_{J}\right)
$$

Where $\hat{\imath}_{C i}$ is the current though the input capacitor of module $\mathrm{j}, \hat{\imath}_{L j}$ is the variation of the inductor current, and $I_{L}$ is the DC current though the inductor.

The addition of expression ( 14) applied to each module results in expressions (15) and (16)

$$
\begin{gathered}
N \hat{\imath}_{I}=\frac{1}{Z_{C}}\left(\hat{v}_{I 1}+\cdots+\hat{v}_{I N}\right)+ \\
+K D \frac{\hat{v}_{O}}{Z_{O}}+\frac{K V_{O}}{R N}\left(\hat{d}_{1}+\cdots+\hat{d}_{N}\right) \\
\hat{\imath}_{I}=\frac{1}{N Z_{C}} \hat{v}_{I F}+\frac{K D}{N} \frac{\hat{v}_{O}}{Z_{O}}+\frac{K V_{O}}{R N^{2}}\left(\hat{d}_{1}+\cdots+\hat{d}_{N}\right)
\end{gathered}
$$

Where $Z_{C}$ is the impedance of the input capacitor (including parasitic resistance) and $R$ is the load resistance.

The input voltage after the filter $\hat{v}_{I F}$ can be related with the input current by means of expression (17).

$$
\hat{v}_{I F}=-\hat{\imath}_{I} \frac{Z_{L f} \cdot Z_{C f}}{Z_{L f}+Z_{C f}}=-\hat{\imath}_{I} Z_{F}
$$

where $Z_{L f}$ and $Z_{C f}$ are the impedances of filter inductor and filter capacitor respectively. $Z_{F}$ is the equivalent impedance of the input filter.

Substituting ( 16) in (15) and operating, the expression of the input current of the modular converter is obtained (19).

$$
\begin{gathered}
\hat{\imath}_{I}=\frac{\frac{V_{O} K}{R}+\frac{V_{G} D K^{2}}{\left(N Z_{O}+Z_{L}\right)} \frac{\left(\hat{d}_{1}+\cdots+\hat{d}_{N}\right)}{N+\frac{Z_{F}}{Z_{C}}+\frac{D^{2} K^{2} Z_{F}}{N Z_{O}+Z_{L}}}}{N} \\
\hat{\imath}_{I}=G_{I d F} \frac{\left(\hat{d}_{1}+\cdots+\hat{d}_{N}\right)}{N}
\end{gathered}
$$

Output voltage can be now expressed as a transfer function depending only on the control signal $\hat{d}_{i}$

$$
\begin{gathered}
\hat{v}_{o}=\frac{K\left(V_{G}-D \frac{\frac{V_{O} K}{R}+\frac{V_{G} D K^{2}}{\left(N Z_{O}+Z_{L}\right)}}{N+\frac{Z_{F}}{Z_{C}}+\frac{D^{2} K^{2} Z_{F}}{N Z_{O}+Z_{L}}} Z_{F}\right)}{N+\frac{Z_{L}}{Z_{O}}} \frac{\left(\hat{d}_{1}+\cdots+\hat{d}_{N}\right)}{N} \\
\hat{v}_{o}=G_{V d F} \frac{\left(\hat{d}_{1}+\cdots+\hat{d}_{N}\right)}{N}
\end{gathered}
$$

Analysis of expression ( 19) and ( 21) establish that from the point of view of total input current and total output voltage, the modular converter can be seen as a single converter where the control signal is the average of control signals of all modules.

\section{B. Input voltage expression derivation}

Expression ( 14) can be modified to obtain the input voltage of a module

$$
\hat{v}_{I j}=\left(\hat{\imath}_{I}-K\left(D \cdot \hat{\imath}_{L j}+I_{L} \cdot \widehat{d}_{J}\right)\right) Z_{C}
$$

The current through the output inductor in module I is

$$
\hat{\imath}_{L j}=\frac{1}{Z_{L}}\left(K D \hat{v}_{I j}+\frac{K V_{G}}{N} d_{j}-\hat{v}_{O}\right)
$$

Substituting (23) in (22), expression (25) is obtained.

$$
\begin{gathered}
\hat{v}_{I j}=\left(\hat{\imath}_{I}-K\left(\frac{V_{O}}{N R}+\frac{D K V_{G}}{N Z_{L}}\right) \hat{d}_{j}-\frac{D^{2} K^{2}}{Z_{L}} \hat{v}_{I j}\right. \\
\left.+\frac{D K}{Z_{L}} \hat{v}_{O}\right) Z_{C} \\
\hat{v}_{I j}=\frac{\left(\hat{\imath}_{I}-K\left(\frac{V_{O}}{N R}+\frac{D K V_{G}}{N Z_{L}}\right) \hat{d}_{j}+\frac{D K}{Z_{L}} \hat{v}_{O}\right)}{\frac{1}{Z_{C}}+\frac{D^{2} K^{2}}{Z_{L}}}
\end{gathered}
$$

Substituting ( 19) and ( 21) in (25) and reordering terms, expression

$$
\begin{gathered}
\hat{v}_{I j}=\frac{G_{I d F}+\frac{D K}{Z_{L}} G_{V d F}}{\frac{1}{Z_{C}}+\frac{D^{2} K^{2}}{Z_{L}}} \frac{\left(\hat{d}_{1}+\cdots+\hat{d}_{N}\right)}{N}- \\
-\frac{K\left(\frac{V_{O}}{N R}+\frac{D K V_{G}}{N Z_{L}}\right)}{\frac{1}{Z_{C}}+\frac{D^{2} K^{2}}{Z_{L}}} \hat{d}_{j} \\
\hat{v}_{I j}=A_{1} \frac{\left(\hat{d}_{1}+\cdots+\hat{d}_{N}\right)}{N}-A_{2} \hat{d}_{j}
\end{gathered}
$$




$$
\begin{gathered}
A_{1}=\frac{G_{I d F}+\frac{D K}{Z_{L}} G_{V d F}}{\frac{1}{Z_{C}}+\frac{D^{2} K^{2}}{Z_{L}}} \\
A_{2}=\frac{K\left(\frac{V_{O}}{N R}+\frac{D K V_{G}}{N Z_{L}}\right)}{\frac{1}{Z_{C}}+\frac{D^{2} K^{2}}{Z_{L}}}
\end{gathered}
$$

The analysis of expression ( 29) shows that the input voltage of every module depends on the average value of control signals, and on the control signal of this particular module.

\section{REFERENCES}

[1] Guanghai Gong, Dominik Hassler, and Johann W. Kolar "A Comparative Study of Multicell Amplifiers for AC-Power-Source Applications" IEEE TRANSACTIONS ON POWER ELECTRONICS, VOL. 26, NO. 11, JANUARY 2011

[2] Raja Ayyanar, Ramesh Giri, and Ned Mohan "Active Input-Voltage and Load-Current Sharing in Input-Series and Output-Parallel Connected Modular DC-DC Converters Using Dynamic Input-Voltage Reference Scheme" IEEE Transactions on Power Electronics, vol. 19, no. 6, pp 1462,1473 Nov 2004

[3] Jung-Won Kim; Jung-Sik Yon; Cho, B. -H, "Modeling, control, and design of input-series-output-parallel-connected converter for highspeed-train power system," Industrial Electronics, IEEE Transactions on , vol.48, no.3, pp.536,544, Jun 2001

[4] Ting Qian; Lehman, B., "Coupled Input-Series and Output-Parallel Dual Interleaved Flyback Converter for High Input Voltage Application," Power Electronics, IEEE Transactions on , vol.23, no.1, pp.88,95, Jan. 2008

[5] Taotao Jin; Zhang, K.; Kan Zhang; Smedley, K., "A New Interleaved Series Input Parallel Output (ISIPO) Forward Converter With Inherent Demagnetizing Features," Power Electronics, IEEE Transactions on , vol.23, no.2, pp.888,895, March 2008

[6] Choudhary, V.; Ledezma, E.; Ayyanar, R.; Button, R.M., "Fault Tolerant Circuit Topology and Control Method for Input-Series and OutputParallel Modular DC-DC Converters," Power Electronics, IEEE Transactions on , vol.23, no.1, pp.402,411, Jan. 2008

[7] X. Ruan, W. Chen, L. Cheng, C. Tse, Hong Yan y Tao Zhang, "Control Strategy for Input-Series-Output-Parallel Converters",IEEE Transactions on Industrial Electronics, vol. 56, no. 4, April 2009.

[8] Y. Huang. "General Control Considerations for Input-Series OutputParallel Converters". IEEE Trans. Ind. Electron., April 2009, VOL. 56, $\mathrm{N}^{\circ} 4$

[9] W. Cheng, X. Ruan, H. Yan, and C.K. Tse, "DC/DC Conversion system consisting of multiple converters modules: stability, control and experimental verification," IEEE Trans. Power. Electron., vol. 24, no. 6, pp. 1463-1474, Jun. 2009.

[10] D. Sha, Z. Guo, and X. Liao, "Cross feedback output current sharing control for input-series-output-parallel modular dc-dc converters," IEEE Trans. Power. Electron., vol. 25, no. 11, pp. 2762-2771, Nov. 2010.

[11] R. Giri, R. Ayyanar, N. Mohan, "Common duty ratio control of input series connected modular DC-DC converters with active input voltage and load current sharing," In Proc. IEEE APEC, 2003, pp.322-326.

[12] Zumel, P.; Ortega, L.; Lazaro, A.; Fernandez, C.; Barrado, A., "Control strategy for modular Dual Active Bridge input series output parallel," in Control and Modeling for Power Electronics (COMPEL), 2013 IEEE 14th Workshop on , vol., no., pp.1-7, 23-26 June 2013

[13] Zumel, P.; Ortega, L.; Lazaro, A.; Fernandez, C.; Barrado, A.; Rodriguez, A.; Hernando, M.M., "Modular dual active bridge converter architecture," in Applied Power Electronics Conference and Exposition
(APEC), 2014 Twenty-Ninth Annual IEEE , vol., no., pp.1081-1087, 1620 March 2014

[14] R.W. Erickson, D. Maksimovic, "Fundamental of Power Electronics", Springer Science \& Business Media, 2007 